\title{
POISSON ANALYSIS AND STATISTICAL MECHANICS
}

\author{
A.L.REBENKO \\ Institute of Mathematics, Ukrainian National Academy of Sciences, \\ 3 Tereshchenkivs'ka St., UA-252601 Kiev, Ukraine
}

Received September 12, 1995

\begin{abstract}
The report presents new Poisson integral representations of grand canonical Gibbs ensemble correlation functions for equilibrium and nonequilibrium continuous systems of classical statistical mechanics. These representations give an opportunity to apply technique, which is well developed for lattice systems. Some new results are announced.
\end{abstract}

\section{Introduction}

In this short report I am going to give some new examples of application of Poisson analysis in physics. There are many well-known examples of application of Gaussian functional integrals for treating models of statistical mechanics. These are the the works by D. C. Brydges and P. Federbush [5], T. Z. Imbrie [2], A. I. Pilyavsky and A. L. Rebenko [3] in which Sine-Gordon transformation was used for Gaussian representation of correlation functions and the work of I. R. Yukhnovskii, M. F. Holovko and their collaborators (see [21]) in which the method of collective variables was applied.

But these methods work only in the case of two-body interaction potential $V(x-y)$, which satisfies the following assumptions:

1.Positive definition: $(f, V f)=\int f(x) V(x-y) f(y) d x d y \geq 0$,

2.Regularity condition: $V(0)<\infty$.

As to assumption 1., a wide class of interactions (including Yukawa and Coulomb interaction with hard-core), does satisfy 1 ., but the second assumption 2. is not accepted from physical point of view as the repulsion forces of two particles should increase to infinity when they collide, i.e. $V(0)=+\infty$.

In the paper [15] a new representation for distribution functions of classical statistical mechanics was proposed. It was based on Poisson measure integral representation. This new form of integral representation for distribution functions gives an opportunity to simplify considerably the construction of cluster expansion and proof of its convergence. In the later work of [16] this method was extended to wide class of interaction potentials, which satisfy only stability condition:

3. $U_{N}\left(x_{1}, \ldots, x_{N}\right) \geq-N B$, and some integrability property.

The main goal of this talk is a short description of this method and announcing some new results, which can be obtained using Poisson measure representation. 
A short contents of this report is following. In Section 2 we briefly state some notions and formulas of the Poisson analysis, needed for later exposition. In Sections 3 and 4 we obtain representations of correlation functions of classical systems for equilibrium and nonequilibrium cases respectively. And finally in the conclusion we enumerate some new results and discuss some perspectives.

\section{Some remarks on the Poisson analysis}

Detailed exposition of different aspects of the Poisson analysis may be found in $[6-11,18]$. In this section we are going to remind only some of the most useful definitions and formulas which will be used later.

\subsection{Definition, support}

Definition 1 We define the Poisson measure $d P_{z}^{\Lambda}$ on the Schwartz space of tempered distributions $S^{\prime}=S^{\prime}\left(\Re^{3}\right)$ by following characteristical functional:

$$
C[\varphi] \equiv \int_{\mathcal{S}^{\prime}} d P_{z}^{\Lambda}(q) e^{i<\varphi, q>}=\exp \left[z \int_{\Lambda}\left(e^{i \varphi(x)}-1\right) d x\right],
$$

where $\varphi \in \mathcal{S}=\mathcal{S}\left(\Re^{\ni}\right), \Lambda \subset \Re^{3},|\Lambda|<\infty$, and $z>0$ is an intensity parameter.

Remark Of course, formula (1) is correct even in case when $\Lambda=\Re^{3}$ but we are going to apply this formalism to the problems of equilibrium statistical mechanics and therefore will start from the finite set $\Lambda$ embedded into $\Re^{3}$.

Notation 1 Let $\mathbf{L}_{2}=L_{2}\left(\mathcal{S}^{\prime},\left\lceil\mathcal{P}_{\ddagger}^{*}\right)\right.$ be the space of functions square integrable with respect to the measure $d P_{z}^{\Lambda}$.

Lemma 1 For any $\varphi(x) \in \mathcal{S}\left(\Re^{\ni}\right)$ the set $\mathbf{E}=\left\{e^{i<\varphi, q>}, \varphi \in \mathcal{S}\right\}$ is dense in $\mathbf{L}_{2}$.

For proof see Theorem 3 in [7].

Notation 2 Let $\delta_{x}=\delta(\cdot-x)$.

Lemma 2 For any $F[q] \in \mathbf{L}_{2}$ the following formula is true:

$$
\int_{S^{\prime}} d P_{z}^{\Lambda}(q) F[q]=e^{-z|\Lambda|} \sum_{n=0}^{\infty} \frac{z^{n}}{n !} \int_{\Lambda^{n}}(d x)_{n} F\left[\sum_{j=1}^{n} \delta_{x_{j}}\right] .
$$

Proof. Let us expand the exponent on the right-hand side of (1) in series, taking into account that $|\Lambda|<\infty$ :

$$
\int_{\mathcal{S}^{\prime}} d P_{z}^{\Lambda}(q) e^{i<\varphi, q>}=e^{-z|\Lambda|} \sum_{n=0}^{\infty} \frac{z^{n}}{n !} \int_{\Lambda^{n}}(d x)_{n} \exp \left[i \sum_{j=1}^{n} \varphi\left(x_{j}\right)\right] .
$$

By Lemma 1 the latter formula may be extended to any $F(q) \in \mathbf{L}_{2}$.

Corollary The set

$$
\Xi_{\Lambda} \equiv\left\{\sum_{j=1}^{n} \delta\left(x-x_{j}\right)\right\}
$$

with Poisson distribution law for $x_{1}, \ldots, x_{n} \in \Lambda\left(x_{i} \neq x_{j}\right.$ if $\left.i \neq j\right)$ is carrie set for the measure $d P_{z}^{\Lambda}(\cdot)$. See [16] for more details. 


\subsection{Formulas for momenta and integrating by parts}

From (1) it follows the next formula for momenta of measure $d P_{z}^{\Lambda}(\cdot)$ :

$$
\begin{aligned}
& \int_{\mathcal{S}^{\prime}} d P_{z}^{\Lambda}(q)<\varphi_{1}, q>\ldots<\varphi_{k}, q>= \\
& =\left.\frac{\partial^{k}}{i \partial \alpha_{1} \ldots i \partial \alpha_{k}} \exp \left[z \int_{\Lambda}\left(\exp \left[i \sum_{j=1}^{k} \alpha_{j} \varphi_{j}(x)\right]-1\right) d x\right]\right|_{\alpha_{1}=\ldots=\alpha_{k}=0}
\end{aligned}
$$

See also [17].

Lemma 3 [13] For any $\varphi \in \mathcal{S}$ and any $F \in \mathbf{L}_{2}$ we have

$$
\int_{\mathcal{S}^{\prime}} d P_{z}^{\Lambda}(q)<\varphi, q>F[q]=z \int_{\mathcal{S}^{\prime}} d P_{z}^{\Lambda}(q) \int_{\Lambda} d x \varphi(x) F\left[q+\delta_{x}\right] .
$$

Proof. As follows from Lemma 1 it is sufficient to prove (4) for $F[q]=$ $\exp [i<\xi, q>], \xi \in \mathcal{S}$ :

$$
\begin{aligned}
\int_{\mathcal{S}^{\prime}} d P_{z}^{\Lambda}(q)<\varphi, q>e^{i<\xi, q>}=\left.\int_{\mathcal{S}^{\prime}} d P_{z}^{\Lambda}(q) \frac{\partial}{i \partial \alpha} e^{i<\xi+\alpha \varphi, q>}\right|_{\alpha=0}, \\
\quad=\left.\frac{\partial}{i \partial \alpha} \int_{\mathcal{S}^{\prime}} d P_{z}^{\Lambda}(q) e^{i<\xi+\alpha \varphi, q>}\right|_{\alpha=0}, \\
\quad=\left.\frac{\partial}{i \partial \alpha} \exp \left[z \int_{\Lambda}\left(e^{i \xi+i \alpha \varphi(x)}-1\right) d x\right]\right|_{\alpha=0}, \\
\quad=z \int_{\Lambda} d x \varphi(x) e^{i \xi(x)} \exp \left[z \int_{\Lambda}\left(e^{i \xi(x)}-1\right) d x\right], \\
=z \int_{\Lambda} d x \varphi(x) e^{i \xi(x)} \int_{\mathcal{S}^{\prime}} d P_{z}^{\Lambda}(q) e^{i<\xi, q>}, \\
=z \int_{\Lambda} d x \varphi(x) e^{i<\xi, q+\delta_{x}>} .
\end{aligned}
$$

\section{3. $U$-transformation and Wick regularization}

Definition 2 As in [6], let us define a U-transformation for a function $F \in \mathbf{L}_{2}$ by the formula:

$$
(U F)(\xi)=C(\xi)^{-1} \int_{\mathcal{S}^{\prime}} d P_{z}^{\Lambda}(q) F[q] e^{i<q, \xi>}, \quad \xi \in \mathcal{S} .
$$

As follows from Lemma 1 , there exists the reverse $U^{-1}$-transformation. It is easy to see from Definitions 1 and 2 that

$$
(U \exp [<\log (1+\varphi), q>])(\xi)=\exp \left[z \int_{\Lambda} e^{i \xi(x)} \varphi(x) d x\right]
$$

and therefore

$$
\left(U^{-1} \exp \left[z \int_{\Lambda} e^{i \xi(x)} \varphi(x) d x\right]\right)(q)=\exp [<\log (1+\varphi), q>] .
$$


Definition 3 Let us define now a Wick regularization by the formula (see [6]):

$$
: F[q]:=\left(U^{-1} F\left[z e^{i \xi}\right]\right)(q) .
$$

From Definition 3 and the formula (7) it directly follows that

$$
: \exp \left[\sum_{j=1}^{k} \alpha_{j}<\varphi_{j}, q>\right]:=\exp \left[<\log \left(1+\sum_{j=1}^{k} \alpha_{j} \varphi_{j}\right), q>\right]
$$

and therefore:

$$
: \prod_{j=1}^{k}\left\langle\varphi_{j}, q>:=\left.\frac{\partial^{k}}{\partial \alpha_{1} \ldots \partial \alpha_{k}} \exp \left[<\log \left(1+\sum_{j=1}^{k} \alpha_{j} \varphi_{j}\right), q>\right]\right|_{\alpha_{1}=\ldots=\alpha_{k}=0}\right.
$$

Remark As can be seen from the latter formula, the Wick regularization coincides with the normal ordering of the Poisson fields regarded in [17].

It immediately follows from (10) and (1) that

$$
\int_{\mathcal{S}^{\prime}} d P_{z}^{\Lambda}(q):<\varphi_{1}, q>\ldots<\varphi_{k}, q>:=z^{k}<\varphi_{1}, 1>\ldots<\varphi_{k}, 1>.
$$

\subsection{The Wick theorems}

The formulas (10) express the Wick polynomials in terms of usual polynomials. If we inverse them we obtain [17] the Poisson analog of well-known in the Quantum field theory and Gaussian analysis Wick theorem [1] which gives the rule of expressing the usual polynomials in terms of the Wick polynomials. To formulate it we need first the following definition:

Definition 4 Let the 'pairing' of the $n$ Poisson fields be the following:

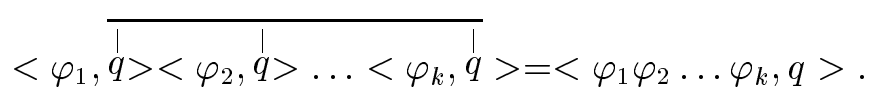

Theorem 1 The usual product of the Poisson fields is equal to the sum of all corresponding normal products with all possible 'pairings' including the normal product without any 'pairing'.

The proof of Theorem 1 uses generalized Wiener-Itô-Segal isomorphism [8] under which the operator of multiplication by the $q(x)$ goes over into the operator $\hat{q}(x)=\left(a^{+}(x)+1\right)\left(a^{-}(x)+1\right)$ on the Fock space $\mathcal{F}=\mathcal{F}\left(\mathcal{L}_{\in}\left(\Re^{\ni}\right)\right)$, where $a^{+}(x)$ and $a^{-}(x)$ are usual creation and annihilation operators:

$$
\left[a^{-}(x), a^{+}\left(x^{\prime}\right)\right]=\delta\left(x-x^{\prime}\right) .
$$

The normal ordering of the product of such operators in the Fock space means their usual product in which all creation operators are placed on the left and annihilation operators are placed on the right. Therefore, after applying Wiener-Itô-Segal isomorphism the proof of Theorem 1 merely turns into a combinatoric exercise (see [17] and [12] for details).

In the same way it may be proved the following generalized Wick theorem which in connection with formula (11) appears to be very useful for calculation of some averages by the measure $d P_{z}^{\Lambda}$. 
Theorem 2 The product of normally ordered products of the Poisson fields is equal to the sum of all corresponding normal products with all possible 'pairings' which connect the Poisson fields from initially different normal products including the normal product without any 'pairing'.

\subsection{Generalized integration by part formula}

To construct the integral representation for distribution functions in statistical mechanics we will also employ the following lemma:

Lemma 4 For any $\Phi, F \in \mathbf{L}_{2}$ and $\varphi \in \mathcal{S}$ the following formula is true:

$$
\int_{\mathcal{S}^{\prime}} d P_{z}^{\Lambda}(q):<\varphi, q>\Phi[q]: F[q]=z \int_{\mathcal{S}^{\prime}} d P_{z}^{\Lambda}(q): \Phi[q]: \int_{\Lambda} d x \varphi(x) F\left[q+\delta_{x}\right]
$$

Proof. Again by virtue of Lemma 1, it is sufficient to prove Lemma 4 for $F[q]=e^{i<\xi, q>}$ and $\Phi[q]=e^{i<\zeta, q>}$, where $\xi, \zeta \in \mathcal{S}$. Using (1) and (9), we have:

$$
\begin{aligned}
& \int_{\mathcal{S}^{\prime}} d P_{z}^{\Lambda}(q):<\varphi, q>e^{i<\zeta, q>}: e^{i<\xi, q>}= \\
& =\frac{\partial}{i \partial \alpha} \int_{\mathcal{S}^{\prime}} d P_{z}^{\Lambda}(q): e^{i<\zeta+\alpha \varphi, q>}:\left.e^{i<\xi, q>}\right|_{\alpha=0}, \\
& \quad=\left.\frac{\partial}{i \partial \alpha} \int_{\mathcal{S}^{\prime}} d P_{z}^{\Lambda}(q) \exp [<\log (1+i \zeta+i \alpha \varphi)+i \xi, q>]\right|_{\alpha=0}, \\
& \quad=\left.\frac{\partial}{i \partial \alpha} \exp \left[z \int_{\Lambda} d x\left(e^{i \xi}(1+i \zeta(x)+i \alpha \varphi(x))-1\right)\right]\right|_{\alpha=0}, \\
& \quad=z \int_{\Lambda} d x e^{i \xi(x)} \varphi(x) \int_{\mathcal{S}^{\prime}} d P_{z}^{\Lambda}(q) \exp [<\log (1+i \zeta)+i \xi, q>] \\
& \quad=z \int_{\Lambda} d x e^{i \xi(x)} \varphi(x) \int_{\mathcal{S}^{\prime}} d P_{z}^{\Lambda}(q): e^{i<\zeta, q>}: e^{i<\xi, q>}, \\
& \quad=z \int_{\mathcal{S}^{\prime}} d P_{z}^{\Lambda}(q): e^{i<\zeta, q>}: \int_{\Lambda} d x \varphi(x) e^{i<\xi, q+\delta_{x}>}
\end{aligned}
$$

\section{Poisson integral representation of the correlation func- tions. Equilibrium case.}

Let us consider the system of classical identical particles contained in a certain finite volume $\Lambda \subset \Re^{3}$ and interacting by $M$-particle $(M \geq 2$ and fixed) potential of general form:

$$
V \equiv\left(V_{2}\left(x_{1}, x_{2}\right), \ldots, V_{M}\left(x_{1}, \ldots, x_{M}\right)\right) .
$$

Then the potential energy for $n$-particles located at points $x_{1}, \ldots, x_{n}$ is:

$$
U(x)_{n}=\sum_{k=2}^{M} \sum_{1 \leq i_{1}<\ldots<i_{k} \leq n} V_{k}\left(x_{i_{1}}, \ldots, x_{i_{k}}\right),
$$

where $(x)_{n}=\left(x_{1}, \ldots, x_{n}\right)$. 
We assume that our potential satisfies the following stability condition:

$$
\exists B \geq 0: \forall(x)_{n} \in \Lambda^{n} \quad U(x)_{n} \geq-B n .
$$

The stability condition allows us to define correlation functions in the grand canonical ensemble as usual:

$$
\rho_{\Lambda}(x)_{m}=\Xi_{\Lambda}^{-1} \sum_{n=0}^{\infty} \frac{z^{m+n}}{n !} \int_{\Lambda^{n}}(d x)_{m+n}^{m+1} \exp \left[-\beta U(x)_{m+n}\right],
$$

where

$$
\Xi_{\Lambda}=\sum_{n=0}^{\infty} \frac{z^{n}}{n !} \int_{\Lambda^{n}}(d x)_{n}^{1} \exp \left[-\beta U(x)_{n}\right]
$$

is the grand partition function, $\beta$ is an inverse temperature and $z$ is an activity.

Theorem 3 Let $\mathbf{V}$ satisfies stability condition (16) then the following formula is valid in the weak sense:

$$
\rho_{\Lambda}(x)_{m}=\Xi(\Lambda)^{-1} \int_{\mathcal{S}^{\prime}} d P_{z}^{\Lambda}(q): q\left(x_{1}\right) \ldots q\left(x_{m}\right): e^{-\beta U_{\Lambda}[q]},
$$

where

$$
U_{\Lambda}[q]=\sum_{p=2}^{M} \frac{1}{p !} \int_{\Lambda^{p}}(d y)_{p}^{1} V_{p}\left(y_{1}, \ldots, y_{p}\right): q\left(y_{1}\right) \ldots q\left(y_{p}\right):
$$

and

$$
\Xi(\Lambda)=e^{-z|\Lambda|} \Xi_{\Lambda}=\int_{\mathcal{S}^{\prime}} d P_{z}^{\Lambda}(q) e^{-\beta U_{\Lambda}[q]} .
$$

Proof. Making renormalization as in (21) and using (2) we can write down $\rho_{\Lambda}$ in the following form:

$$
\rho_{\Lambda}(x)_{m}=z^{m} \Xi(\Lambda)^{-1} \int_{\mathcal{S}^{\prime}} d P_{z}^{\Lambda}(q) e^{-\beta U[q](x)_{m}},
$$

where

$$
\begin{aligned}
U[q](x)_{m}= & \sum_{0 \leq k \leq p \leq M} \frac{1}{k !} \sum_{1 \leq i_{1}<i_{2}<\ldots<i_{p-k} \leq m} \int_{\Lambda^{k}}(d y)_{k}^{1} \\
& \times V_{p}\left(x_{i_{1}}, \ldots, x_{i_{p-k}}, y_{1}, \ldots, y_{k}\right): q\left(y_{1}\right) \ldots q\left(y_{k}\right):
\end{aligned}
$$

with $V_{0}=V_{1}=0$.

It is easy to see that lemma 4 applied $m$-times to (22) gives (19).

\section{Poisson integral representation of the correlation func- tions. Nonequilibrium case}

Following the paper [20] we define the following characteristical functional:

$$
C_{\rho}^{t}(\eta)=\sum_{N=0}^{\infty} \int(d x)^{N} \rho_{N}\left(x_{1}, \ldots, x_{N}\right) e^{i \sum_{j=1}^{N} \eta\left(x_{j}\right)},
$$


where $\rho_{N} \geq 0$ and

$$
\sum_{N=0}^{\infty} \frac{z^{N}}{N !} \int(d x)^{N} \rho_{N}\left(t, x_{1}, \ldots, x_{N}\right)=1 .
$$

Here $x=(q, p) \in \Re^{6}$ is a point in the coordinate-momentum phase space. Then from Minlos theorem

$$
C_{\rho}^{t}(\eta)=\int_{S^{\prime}} e^{i(\eta, \sigma)} d \mu_{\rho}^{z, t}(\sigma) .
$$

Now we consider the case for which a function $E_{\Lambda}^{t} \in L^{2}\left(d P_{z}^{\Lambda}(\sigma)\right)$ exists so that

$$
E_{\Lambda}^{t}\left(\sum_{j=1}^{N} \delta\left(\cdot-x_{j}\right)\right)=\rho_{N}^{\Lambda}\left(t, x_{1}, \ldots, x_{N}\right)=e^{z|\Lambda|} \rho_{N}\left(t, x_{1}, \ldots, x_{N}\right) .
$$

Then using (2) we have

$$
C_{\rho}^{t}(\eta)=\int d P_{z}^{\Lambda}(\sigma) E_{\Lambda}^{t}(\sigma) e^{i(\eta, \sigma)} .
$$

So from (27) and (29) we conclude that measure $d \mu_{\rho}^{z}(\sigma)$ is absolutely continuous with respect to Poisson measure $d P_{z}^{\Lambda}(\sigma)$ for $\rho_{N}$ which is given by (28):

$$
d \mu_{\rho}^{z}, t(\sigma)=E_{\Lambda}^{t}(\sigma) d P_{z}^{\Lambda}(\sigma) .
$$

Then we assume that $\rho_{N}\left(t, x_{1}, \ldots, x_{N}\right)$ is the solution to the Liouville equation:

$$
\frac{\partial \rho_{N}^{\Lambda}\left(t, x_{1}, \ldots, x_{N}\right)}{\partial t}=\left\{H_{N}, \rho_{N}^{\Lambda}\right\}
$$

where

$$
H_{N}=\sum_{j=1}^{N} \frac{p_{j}^{2}}{2 m}+\sum_{1 \leq i \leq N} V\left(q_{i}-q_{j}\right)
$$

is the Hamiltonian of $N$-particle and $\{\cdot, \cdot\}$ is Poisson bracket.

It is well-known that nonequilibrium distribution functions which satisfy BBGKY chain equations can be constructed from $\rho_{N}^{\Lambda}\left(t, x_{1}, \ldots, x_{N}\right)$ according to the formula

$$
f_{N}^{\Lambda}\left(t, x_{1}, \ldots, x_{N}\right)=\sum_{m=0}^{\infty} \frac{z^{m+N}}{m !} \int_{\left(\Lambda \otimes R^{3}\right)^{m}} d x_{N+1} \ldots d x_{N+m} \rho_{N+m}^{\Lambda}\left(t, x_{1}, \ldots, x_{N}\right) .
$$

Using (33), (25) - (27) and (9) we get the representation for generating functional of distribution functions $f_{N}^{\Lambda}\left(t, x_{1}, \ldots, x_{N}\right)$ :

$$
\begin{aligned}
F\{t, j\} & =\sum_{N=0}^{\infty} \frac{1}{N} \int d x_{1} \ldots d x_{N} f_{N}^{\Lambda}\left(t, x_{1}, \ldots, x_{N}\right) j\left(x_{1}\right) \cdots j\left(x_{N}\right)= \\
& =\int_{S^{\prime}} d \mu_{\rho}^{z, t}(\sigma) e^{(\sigma, \log [1+j(\cdot)])}= \\
& =\int_{S^{\prime}} d \mu_{\rho}^{z, t}(\sigma): e^{(\sigma, j)}:
\end{aligned}
$$

The next step is the following lemma (see [20]) 


\section{Lemma 5}

$$
\begin{aligned}
F\{t, j\} & =\int e^{\left(U_{t} \sigma, \log [1+j(\cdot)]\right)} d \mu_{r} h o^{z, 0}= \\
& =\int: e^{\left(U_{t}, j\right): d \mu_{r} h o^{z, 0}}
\end{aligned}
$$

where $U_{t} \sigma=\sigma(t, x)$ satisfies the Vlasov equation

$$
\frac{\partial \sigma(t, x)}{\partial t}=-\frac{p_{(\nu)}}{m} \frac{\partial \sigma(t, x)}{\partial q^{(\nu)}}+\frac{\partial \sigma(t, x)}{\partial p^{(\nu)}} \int d x^{\prime} \sigma\left(t, x^{\prime}\right) \frac{\partial V\left(q-q^{\prime}\right)}{\partial q^{(\nu)}}
$$

with initial date $\sigma(0, x)=\sigma(x)$.

The proof follows from direct verification that (35) satisfies BBGKY functional equation for $F(t, j)$ if $(36)$ is valid.

So from (35) we have

$$
f_{m}\left(t, x_{1}, \ldots, x_{m}\right)=\int d \mu_{\rho}^{z, 0}(\sigma): \sigma\left(x_{1}, t\right) \cdots \sigma\left(x_{m}, t\right): .
$$

Now using (30) and choosing initial data for Liouville equation as perturbation of stationary distribution by potential $w(q)$ we obtain the following representation:

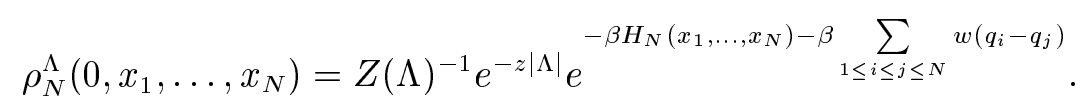

\section{Conclusion}

Using introduced representation, we can compose cluster expansion for systems with many-body interaction and prove its convergence (see [19]) in spite of the remarks of D.Bryges [4]. We also state that in nonequilibrium case we can form cluster expansion for (38) and apply the well-developed method of cluster expansion. This work is our nearest perspectives.

\section{References}

[1] Bogolubov N.N., Shirkov D.V. Introduction to the theory of quantized fields. New York, Interscience, 1959.

[2] Imbrie T.Z. Debye screening for helium and other Coulomb systems. // Communications in Mathematical Physics, 1983, vol. 87, p. 515-565.

[3] Pilyavsky A.I., Rebenko A.L. Debye screening in spatially inhomogeneous systems of charged particles. I. A model of spherical insulator. II. A proof of convergence of cluster expansions. // Teor. Mat. Fiz., 1986, vol. 69, p. 245-258; 1987, vol. 70, p. 278-288.

[4] Bridges D.C. A short course of cluster expansion. Les Houches, Session XLIII, 1984 .

[5] Bridges D.C., Federbush P. Debye screening in dilute classical Coulomb systems. // Communications in Mathematical Physics, 1980, vol. 73, p. 197-246.

[6] Ito Y. On a generalization of non-linear Poisson functionals. // Math. Rep. Toyoma Univ., 1980, vol. 3, p. 111-122.

[7] Ito Y. Generalized Poisson functionals. // Probability Theory and Related Fields, 1988, vol. 77, p. 1-28. 
[8] Ito Y., Kubo I. Calculus on Gaussian and Poisson white noises. // Nagoya Mathematical Journal, 1988, vol. 111, p. 41-84.

[9] Kabanov Ju.M. On extended stochastic integrals. // Probability Theory and Application, 1975, vol. 20, No 4, p. 725-737 (in Russian).

[10] Kallenberg O. Lecture on random measures. University of North Carolina, 1974.

[11] Kerstan J., Matthes K., Mecke J. Unbegrenzt teilbare Punktprozesse. Berlin, Akademie-Verlag, 1974.

[12] Lytvynov E.W., Rebenko A.L., Shchepan'uk G.V. Wick theorems in nonGaussian white noise calculus (to appear in Reports in Mathematical Physics).

[13] Mecke I. Stationäre zufällige Maße auf localkompakten Abelschen Gruppen. // Zeitschr. für Wahrscheinlichkeitstheorie verw. Gebiete, 1967, vol. 9, p. 3658.

[14] Nguyen X.X., Zessin H. Integral and differential characterizations of the Gibbs process. // Mathematische Nachrichten, 1979, vol. 88, p. 105-115.

[15] Rebenko A.L. Poisson measure representation and cluster expansion in classical statistical mechanics. // Communications in Mathematical Physics, 1993, vol. 151, p. 427-443.

[16] Gielerak R., Rebenko A.L. On the Poisson integrals representation in the classical statistical mechanics of continuous systems. Preprint BiBoS No 633/5/94, Bielefeld, 1994.

[17] Shchepan'uk G.V. Poisson fields and distribution functions in statistical mechanics of charged particles. // Ukr. Mat. Zhurn., 1995, vol. 47, No 5, p. 710-719 (in Ukrainian).

[18] Surgalis D. On multiple stochastic integrals and associated Marcov semigroups. // Probability and Mathematical Statistics, 1984, vol. 3, No 2, p. $217-239$.

[19] Rebenko A.L., Shchepan'uk G.V. The convergence of cluster expansion for continuous systems with many-body interaction. Preprint BiBoS No 690/6/95, Bielefeld, 1995. (submitted to Journal of Statistical Physics).

[20] Maslov V.P., Chebotarev A.M. On random fields corresponding to the Bogolubov, Vlasov and Boltzmann chains. // Teor. Mat. Fiz., 1983, vol. 54, p. 78-88 (in Russian).

[21] Yukhnovskii I.R., Holovko M.F. Statistical theory of classical equilibrium systems. Kiev, Naukova Dumka, 1980 (in Russian).

\section{ПУАССОНІВСЬКИЙ АНАЛІЗ ТА СТАТИСТИЧНА МЕХАНІКА}

\section{А.Л.Ребенко}

В доповіді приведене нове представлення корреляційних функцій великого канонічного ансамблю для рівноважних та нерівноважних неперервних систем класичної статистичної механіки. Ці представлення дають можливість застосувати техніку, що добре розроблена для гратчатих систем. Анонсуються деякі нові результати. 